\title{
Electron- and hole-phonon interaction in quantum dot embedded into semiconductor medium (GaAs/Al $\left.\mathrm{Ga}_{1-x} \mathrm{As}\right)$
}

\author{
M.V.Tkach, M.Y.Mikhalyova, O.M.Voitsekhivska, \\ R.B.Fartushinsky \\ Chernivtsi State University, \\ 2 Kotsiubinsky Str., 274012 Chernivtsi, Ukraine
}

Received May 24, 2001

\begin{abstract}
The analytical and numerical calculations of electron and hole spectra renormalised by L- and I-phonons taking into account the configurational interaction are performed for the QD embedded into semiconductor medium exemplified by $\mathrm{GaAs} / \mathrm{Al}_{x} \mathrm{Ga}_{1-x}$ As nanoheterosystems.

It is established that for the nanosize QDs the shifts of electron and hole ground levels are created by the interaction of these quasiparticles with Land I-phonons due to all the states of discrete and continuous spectrum. For the small QDs, the shifts of ground energy levels have strong nonlinear dependences while for the big QDs, they almost do not depend on QD radius and have the magnitude close to the shifts of ground levels in massive crystal creating QD. Due to the different effective masses of light and heavy holes, the splittings of their ground levels are the complicated functions on $\mathrm{QD}$ radius and $\mathrm{Al}$ concentration in $\mathrm{Al}_{x} \mathrm{Ga}_{1-x}$ As medium.
\end{abstract}

Key words: nanoheterosystem, interaction, electron, hole, phonon

PACS: $79.60 . J v, 63.20 . D j$

\section{Introduction}

Numerous theoretical and experimental investigations [1-3] during the past decade are devoted to the theory of electron-phonon interaction in low-dimensional systems. Nevertheless, the problem of spectral parameters dependence on the geometrical parameters of nanosystems is discussed up till now. Different physical models and mathematical approximations [4] are used for the description of the above mentioned systems.

It is known [4] that the main model for studying the electron-phonon interaction in the simplest heterosystems (plane quantum wells [5-6], quantum wires (QW) [7- 
8] and quantum dots (QD) [9-12]) is the dielectric continuum model. It gives rather exact results compared to the Huang-Zhu microscopic model [4]. The calculation of electron (hole) energy renormalised due to the interaction with phonons is a sophisticated mathematical problem even within the framework of dielectric continuum model. The reasons of these difficulties are the multilevel and multiband electron (hole) spectrum and the presence of several modes of phonon spectrum.

In order to simplify the problem some authors [9-10] considered only the interlevel interaction between electron and optical phonons assuming the other types of interaction as secondary. At this approximation, the interlevel interaction through the continuous states is not taken into account, as well as the interaction with interface phonons which are absent in spherical QDs only for the spherically-symmetric states $(l=0)$. It is true only for the small QDs when there is a ground energy level in the potential well or the excited levels are located quite far away. But in [13] it was shown that for the plane QWs the interlevel interaction becomes essential for the big QWs. When the QW width increases from zero to the infinity, the shift of the band bottom and the electron effective mass smoothly vary in the limits close to the corresponding three-dimensional magnitudes.

The other group of authors [7, 11-12] have used the approximation of infinitely deep potential well in the media interface, justifying such mathematical simplification by the small difference between electron wave functions in the potential well of infinite and finite depth. Herein, there is not taken into account the shift of the external medium phonons which is rather big for the small radii of a heterosystem and the real shift of interface phonons is essentially smaller as well.

In this paper the different types of electron, light and heavy hole interaction with optical and interface phonons through the discrete and continuous states of GaAs spherical QD embedded into $\mathrm{Ga}_{x} \mathrm{Al}_{1-x} \mathrm{As}$ massive external medium are investigated in detail.

\section{Electron-phonon Hamiltonian in spherical nanohetero- system}

The renormalization of the electron (hole) ground level due to the interaction with phonons is under study for the semiconductor QD embedded into a massive semiconductor sphere. According to the general theory [14], the Hamiltonian of electron interacting with phonon in the representation of the second quantization over all the variables of nanoheterosystem has the following form

$$
\hat{H}=\hat{H}_{e}+\hat{H}_{L}+\hat{H}_{I}+\hat{H}_{e-L}+\hat{H}_{e-I},
$$

where

$$
\hat{H}_{e}=\sum_{p l m} E_{p l} \hat{a}_{p l m}^{+} a_{p l m}
$$

is the Hamiltonian of the electron subsystem. Here $p$ is the set of two radial quantum numbers $(n, k)$ denoting the states of discrete and continuous spectra, respectively; 
$l, m$ are orbital and magnetic quantum numbers. The discrete spectrum energies $E_{n l}$ are defined by the solutions of the dispersion equations [14] and the continuous spectrum energies are fixed by the expression

$$
E_{k l}=\frac{\hbar^{2} k^{2}}{2 m_{1}}
$$

where $m_{1}$ is the quasiparticle effective mass in the external medium.

$$
\begin{aligned}
\hat{H}_{L} & =\sum_{i=0}^{1} \sum_{s_{i} l m} \Omega_{L i}\left(\hat{b}_{s_{i} l m}^{+} \hat{b}_{s_{i} l m}+\frac{1}{2}\right), \\
\hat{H}_{I} & =\sum_{l m s= \pm} \Omega_{l}^{(s)}\left(\hat{b}_{s l m}^{+} \hat{b}_{s l m}+\frac{1}{2}\right)
\end{aligned}
$$

are the Hamiltonians of confined and interface phonons, respectively. The energies of confined phonons $\left(\Omega_{L_{i}}\right)$ are equal to the corresponding energies of the longitudinal optical vibrations of the massive crystals, and the energies of interface phonons $\left(\Omega_{l}^{(s)}\right)$ are given by the dispersion equations [14]. Index $s_{i=0,1}$ corresponds to the radial quantum numbers of the confined polarizational vibrations and $s= \pm-$ numerates two modes of interface vibrations.

$$
\begin{aligned}
\hat{H}_{e-L}= & \sum_{i=0}^{1} \sum_{\substack{p_{1} l_{1} m_{1} \\
p_{2} l_{2} m_{2}}} \sum_{s_{i} l m} \Phi_{p_{1} l_{1} m_{1}}^{p_{2} l_{2} m_{2}}\left(s_{i} l m\right) \hat{a}_{p_{2} l_{2} m_{2}}^{+} \hat{a}_{p_{1} l_{1} m_{1}}\left(\hat{b}_{s_{i} l m}^{+}+\hat{b}_{s_{i} l-m}\right), \\
\hat{H}_{e-I}= & \sum_{\substack{p_{1} l_{1} m_{1} \\
p_{2} l_{2} m_{2}}} \sum_{s l m} \Phi_{p_{1} l_{1} m_{1}}^{p_{2} l_{2} m_{2}}(s l m) \hat{a}_{p_{2} l_{2} m_{2}}^{+} \hat{a}_{p_{1} l_{1} m_{1}}\left(\hat{b}_{s l m}^{+}+\hat{b}_{s l-m}\right)
\end{aligned}
$$

are the Hamiltonians of interaction between electron and L-, I-phonons, respectively. The expressions for the binding functions $\Phi_{p_{1} l_{1} m_{1}}^{p_{2} l_{2} m_{2}}\left(s_{i} l m\right)$ and $\Phi_{p_{1} l_{1} m_{1}}^{p_{2} l_{2} m_{2}}(s l m)$ are presented in [14].

Finally, the Hamiltonian given by equation (1) makes it possible to use the Greens function method for the investigation of electron (hole) spectrum renormalized due to the interaction with phonons.

\section{Calculation and analysis of the ground electron level renor- malization due to the interaction with optical and interface phonons}

It is known [15-16], that in the case of QD with multilevel electron spectrum, the Fourier image of Green's function is connected with mass operator (MO) of the system by Dyson equation

$$
G_{\mu \mu^{\prime}}(\omega)=G_{\mu \mu^{\prime}}^{0}(\omega) \delta_{\mu \mu^{\prime}}+G_{\mu}^{0}(\omega) \sum_{\mu_{1}} M_{\mu \mu^{\prime}} G_{\mu_{1} \mu^{\prime}}(\omega)
$$


Table 1. Values of physical parameters used in numerical calculations.

\begin{tabular}{|c|c|c|}
\hline & GaAs & $\mathrm{Al}_{x} \mathrm{Ga}_{1-x} \mathrm{As}$ \\
\hline$m_{e}$ & 0.067 & $0.067+0.083 x$ \\
\hline$m_{l h}$ & 0.08 & $0.08+0.1 x$ \\
\hline$m_{h h}$ & 0.035 & $0.35+0.05 x$ \\
\hline$\epsilon_{0}$ & 10.6 & $10.9-2.8 x$ \\
\hline$\epsilon_{\infty}$ & 12.5 & $13.2-3.1 x$ \\
\hline$\Omega_{L o}(\mathrm{meV})$ & 36.2 & 50.1 \\
\hline$E_{g}$ & 1.42 & $1.42+1.155 x+0.37 x^{2}$ \\
\hline
\end{tabular}

where

$$
G_{\mu}^{0}(\omega)=\left(\omega-E_{\mu}+\mathrm{i} \eta\right)^{-1}
$$

and $\mu=n, l, m$ is the set of all quantum numbers characterizing the electron states of the discrete spectrum.

According to [15], in case of the weak binding which is realised for the researched $\mathrm{GaAs} / \mathrm{Al}_{x} \mathrm{Ga}_{1-x} \mathrm{As}$ nanoheterosystem, the MO describing the ground state $(\mu=$ 100) renormalization, has the form

$$
M_{100,100}(\omega)=M(\omega)=\sum_{i=o}^{1} \sum_{p l m} \frac{\sum_{S_{i}}\left|\left(F_{S_{i}}\right)_{10}^{p l}\right|}{\omega-E_{p l}-\Omega_{L_{i}}}+\sum_{s p l m} \frac{\sum\left|\left(F_{l}^{(s)}\right)_{10}^{p l}\right|}{\omega-E_{p l}-\Omega_{l}}
$$

where $E_{p l}, \Omega_{L_{i}}, \Omega_{l}^{(s)}$ are the energies of electron and phonon subsystems and $\left(F_{S_{i}}\right)_{10}^{p l}$, $\left(F_{l}^{(s)}\right)_{10}^{p l}$ are the radial parts of binding functions of these quasiparticles obtained in $[14]$.

The pole of the Greens function Fourier image brings to the dispersion equation defining the energy $\left(\tilde{E}_{10}\right)$ of the electron ground level renormalized due to phonons as

$$
\tilde{E}_{10}=E_{10}+\Delta
$$

where according to the MO structure (10) the shift $\Delta$ is given by the sum of partial shifts

$$
\Delta=\Delta_{L_{0} d}+\Delta_{L_{1} d}+\Delta_{I^{+} d}+\Delta_{I^{-} d}+\Delta_{L_{0} c}+\Delta_{L_{1} c}+\Delta_{I^{+} c}+\Delta_{I^{-} c}
$$

due to the respective phonon modes through the states of discrete (d) and continuous (c) parts of electron spectrum.

The numerical calculation of electron, light and heavy holes ground level total and partial shifts was performed for the QD with physical parameters listed in table 1 . The dependences of the electron and hole ground level total and partial shifts on QD radius are qualitatively the same, and therefore, the analysis is performed for the electron only.

Figures 1-4 present the total and partial shifts of electron ground level (in dimensionless units of optical phonon energy of GaAs crystal) determined by different 
types of interaction with phonons as functions of $\mathrm{QD}$ radius (in units of GaAs lattice constant).

Figure 1a shows the picture of the formation of GaAs QD and phonons of the $\mathrm{Al}_{x} \mathrm{Ga}_{1-x}$ As external medium between the electron and the confined optical phonons through all the discrete states (d). Namely: $\Delta_{L_{1} d}^{e}$ is the shift produced by the interaction with phonons of the external medium, $\Delta_{L_{0} d}^{e}(n l)$ - by phonons of internal medium through the $n l$-th state, $\sum_{n l}^{\prime} \Delta_{L_{0}}^{e}(n l)$ - by phonons of internal medium through all excited states, $\Delta_{L d}^{e}$ - the sum partial shift due to the interaction with the confined phonons through all the states of a discrete spectrum. From the figure it is clear that when the energy level appears in GaAs well, its shift is formed by the intralevel interaction $\Delta_{L_{0} d}^{e}(10)+\Delta_{L_{1} d}^{e}$. When the QD radius $\left(r_{0}\right)$ increases, the partial shift $\Delta_{L_{0} d}^{e}$ produced by the intralevel interaction with $L_{o}$-phonons, reaches its maximum and then slowly decreases. For the big QD there are the excited electron states in the well $(n \neq 1, l \neq 0)$. Their interaction with phonons gives the corresponding partial shifts $\Delta_{L_{0} d}^{e}(n l)$. The dependences of these shifts on $r_{0}$ are qualitatively the same - after the appearance of the respective state in the well their magnitude smoothly increases, reaches their maximum and then slowly decreases. After the appearance of every new bound states in QD, the absolute magnitude of the partial shifts becomes smaller. Thus, for the fixed QD radius the shift produced by the intralevel interaction is bigger than the shift formed due to every excited state. But for rather big sizes of the internal medium there is a big number of energy levels in GaAs well, thus the sum shift due to interlevel interaction is much bigger than the intralevel one. The interlevel interaction becomes more essential for the bigger QD radii. It increases at bigger $r_{0}$, reaches the magnitude of intralevel interaction and then becomes a basic one.

In figure 1a the dependence of $\Delta_{L d}^{e}$ partial shift on $r_{0}$ is shown by a dashed curve. It is clear that this therm is essential only for the small QD radii and has a sharp minimum vanishing at $r_{0} \geqslant 20 a_{\mathrm{GaAs}}$. Such a behaviour of the curve is explained by the fact that only for the small QDs the probability of electron location in $\mathrm{Al}_{x} \mathrm{Ga}_{1-x} \mathrm{As}$ barrier is bigger than the probability of its location in GaAs well. The $\Delta_{L d}^{e}$ magnitude is comparable to the $\Delta_{L_{0} d}^{e}(10)$. When the QD radius increases, the electron is "involved" into GaAs which corresponds to the vanishing of $\Delta_{L_{1} d}^{e}$ shift, formed by the phonons of the external medium.

In figure $1 \mathrm{~b}$ the partial shifts caused by the interaction of electron with interface phonons through all the states of discrete spectrum are presented as the functions of QD radius. Namely: $\Delta_{I^{ \pm}}^{e}(n l)$ is the shift produced by $I^{ \pm}$-phonons through the $n l$ th-state, $\Delta_{I^{ \pm} d}^{e}$ is the shift produced by $I^{ \pm}$-phonons through all the excited states, $\Delta_{I d}^{e}$ is total partial shift due to the interaction with interface phonons through all the states of discrete spectrum.

Thus in the central-symmetric states $(l=0)$ there are no interface phonons $[9,14]$, it is natural that in the figure, the shifts are presented formed by the interlevel interaction only. Thus, the shifts caused by interface phonons start to arise at such QD radius when the first excited level appears in the well. It is clear that for the arbitrary radius of the internal medium the partial shifts $\Delta_{I^{+} d}^{e}$ (dotted curve) are 

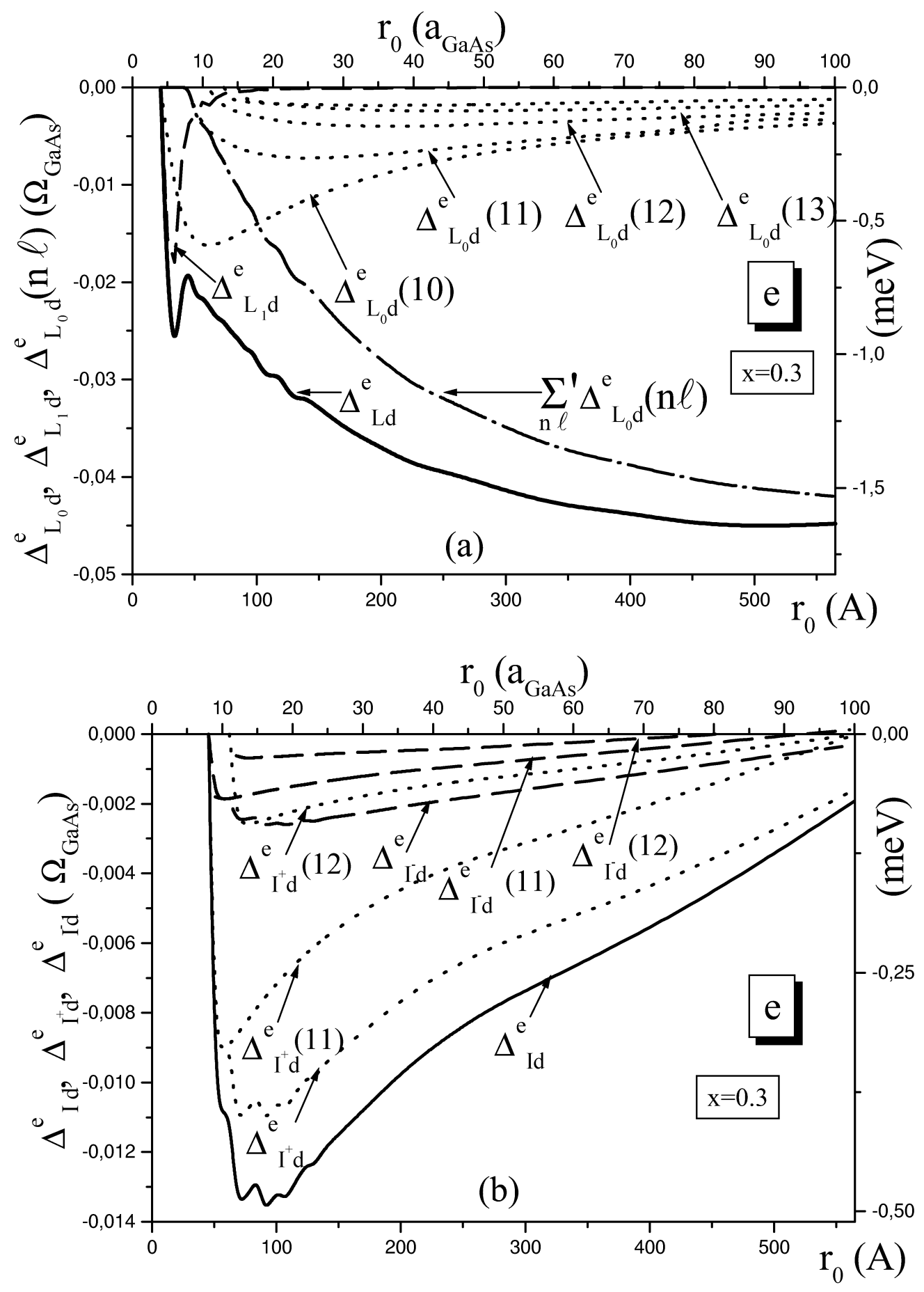

Figure 1. Dependences of partial shifts caused by electron interaction with confined (a) and interface (b) phonons through the discrete states on QD radius. 


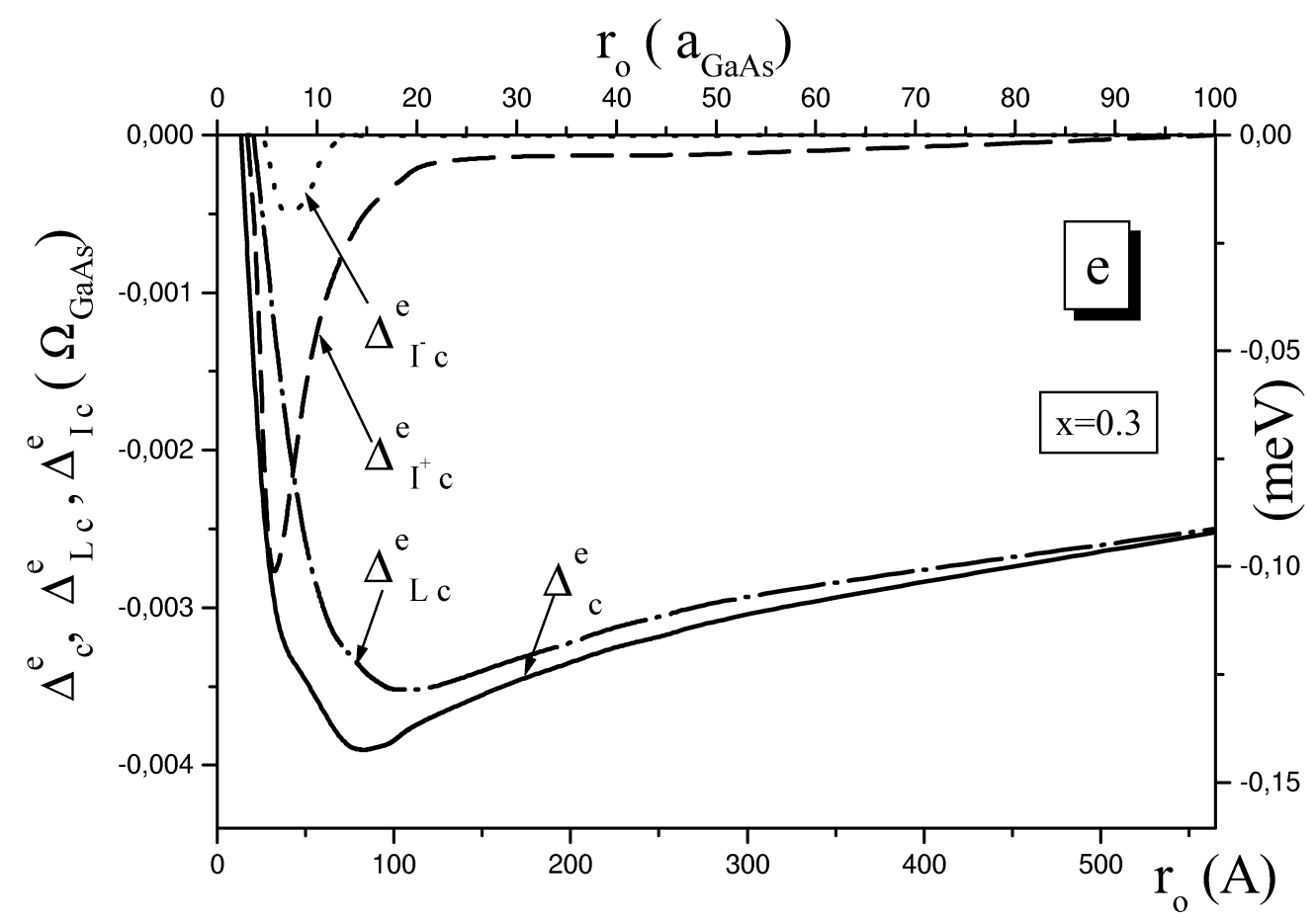

Figure 2. Dependences of partial shifts caused by electron interaction with confined (a) and interface (b) phonons through the continuous states on QD radius.

bigger than $\Delta_{I^{-} d}^{e}$ (dashed curve). When $r_{0}$ increases, the partial shift of every level, after its appearance, rapidly increases to its maximum magnitude and then slowly vanishes. Therefore, the total shift caused by the interaction with interface phonons reaches its maximum at $r_{0} \approx 15$ and then slowly decreases.

In figure 2 the picture of the formation of ground electron level shift caused by the interaction with all phonons through all the continuous states is presented. From the figure one can see that when the QD radius increases the partial shift of L-phonons into $\Delta_{L c}^{e}$ magnitude slowly decreases after reaching the maximum. The partial shifts $\Delta_{I^{+} c}^{e}$ and $\Delta_{I^{-}{ }_{c}}^{e}$ are formed at such QD radius, when it appears that the dependence of these partial shifts on $r_{0}$ is qualitatively the same but quantitatively $\left|\Delta_{I^{+}{ }_{c}}^{e}\right|$ is several times bigger than $\left|\Delta_{I^{-} c_{c}}^{e}\right|$ at any $r_{0}$ values.

The reason of sharp minimum in $\Delta_{I^{ \pm} d}^{e}$ curve lies in the fact that the magnitude of electron-interface phonons interaction is proportional to the density of probability of electron location in the vicinity of media interface, where the potential of I-phonon field has its maximum. When the QD radius increases, the probability reaches its maximum (the electron is "involved" from the barrier into the well) and the interaction with interface phonons becomes bigger. At further increasing of the radius the probability vanishes and, as a result, $\left|\Delta_{I^{ \pm} d}^{e}\right|$ decreases.

In figures 3a,b the total $\left(\Delta^{e, h}\right)$ and partial $\left(\Delta_{L}^{e, h}, \Delta_{I}^{e, h}\right)$ shifts produced due to the electron (figure $3 \mathrm{a}$ ) and hole (figure $3 \mathrm{~b}$ ) interaction with confined (L) and interface (I) phonons through all the states of discrete spectrum are shown as functions of QD radius. The partial shifts $\Delta_{l, I c}^{e, h}$, caused by the interaction with all phonons through 

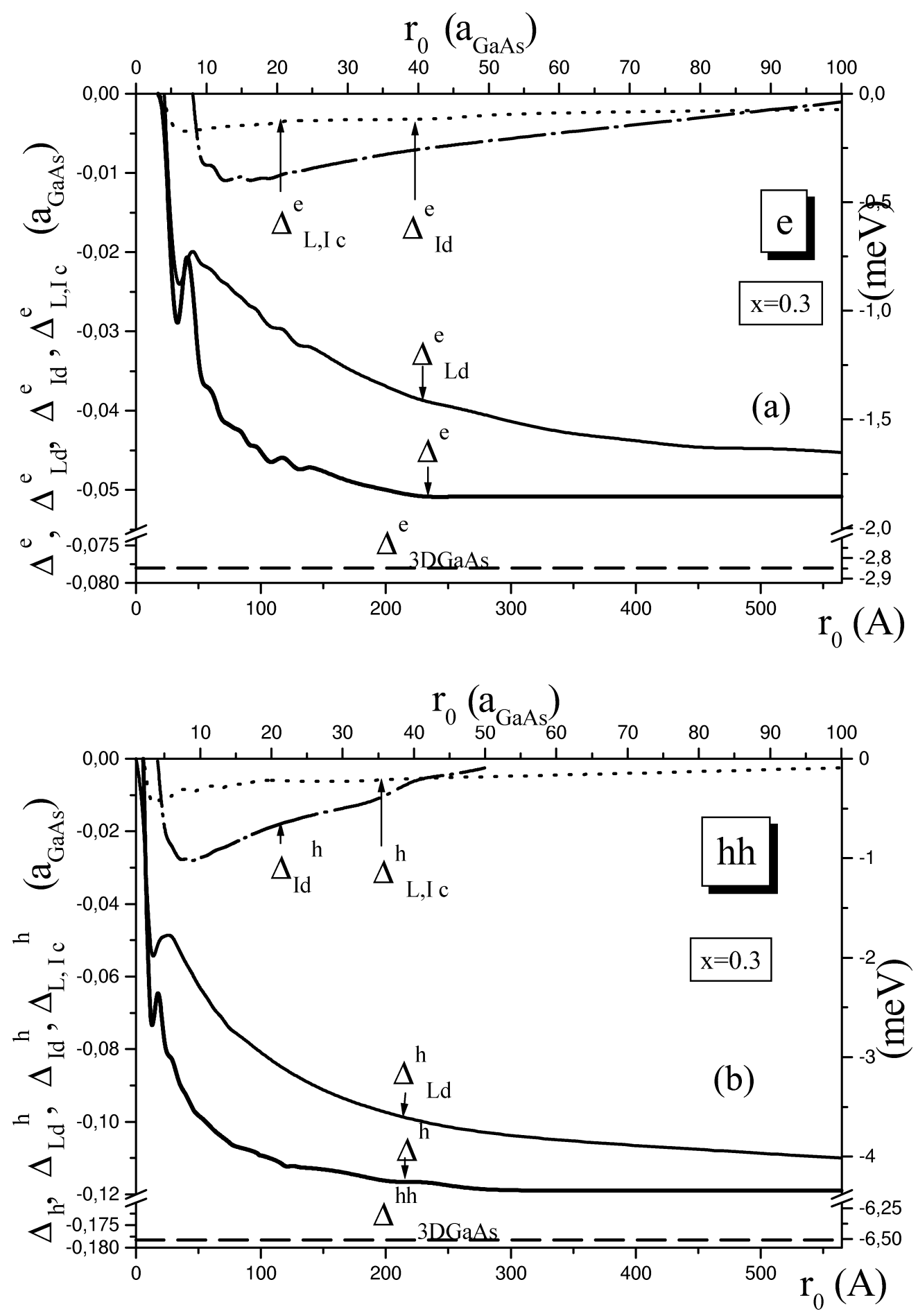

Figure 3. Dependences of partial shifts of ground electron (a) and heavy hole (b) energy levels due to the interaction with interface and confined phonons on QD radius. 


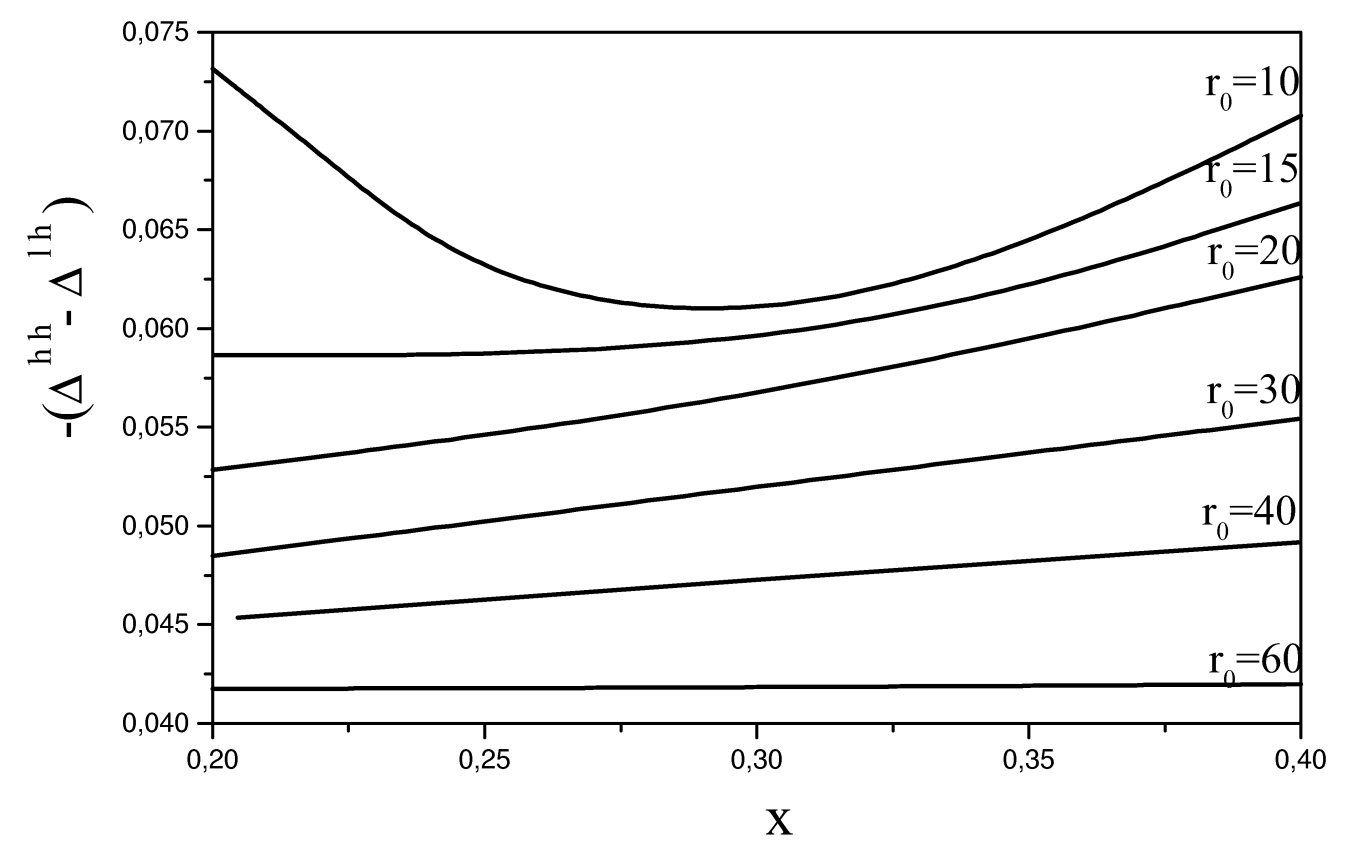

Figure 4. Dependence of splitting between total shifts of light and heavy holes on $\mathrm{QD}$ radius.

the states of continuous (c) spectrum are presented as well. The light hole has the effective mass close to the mass of electron, since the behaviour of its total and partial shifts is almost the same as for the electron.

In the curve $\left(\Delta^{e}\right)$ in the vicinity $r_{0}=4$ one can see the sharp minimum produced by the interaction with the confined phonons of the external medium $\left(L_{1}\right)$. Further, the curve smoothly limits to the saturation. The shift $\left(\Delta_{I d}^{e}\right)$, formed by the interaction with interface phonons through the discrete states, tends to vanish. As a result, the total shift depends on $r_{0}$ only for small QD radii. For the big QDs the magnitude of the shifts is close to its value $\left(\Delta_{\mathrm{GaAS}}^{3 D e, h h, l h}\right)$ in an analogue massive crystal creating the QD. The small difference between these shifts arises due to the neglecting of non-diagonal terms of higher order MO.

Figure $3 \mathrm{~b}$ proves that the behaviour of heavy hole shifts is qualitatively equivalent to the behaviour of electron shifts.

Due to the difference between light and heavy hole effective masses, the interaction with the phonons eliminates the degeneration of these quasiparticles energies in ground states. The magnitude of splitting $\left(D_{h}\right)$ of light and heavy hole ground levels is determined by the difference of their shifts

$$
D_{h}=E^{h h}-E^{l h}=\Delta^{h h}-\Delta^{l h}
$$

thus $D_{h}$, in general case, depends on the radius and on $\mathrm{Al}$ concentration $(x)$ in a nanosystem.

In figure 4, the dependence of $D_{h}$ splitting on $\mathrm{Al}$ concentration (x) in $\mathrm{Al}_{x} \mathrm{Ga}_{1-x} \mathrm{As}$ medium is shown for the different QD radii $\left(r_{0}\right)$. From the figure one can see that for the small QDs $\left(r_{0}<15 a_{\mathrm{GaAs}}\right)$, the $D_{h}(x)$ dependence is nonlinear, for the big 
QDs $\left(15 a_{\mathrm{GaAs}}<r_{0}<20 a_{\mathrm{GaAS}}\right)$ it is linear and at further $r_{0}$ increasing, $D_{h}$ does not depend on $\mathrm{x}$. It is caused by the fact that the shifts are very sensitive (strongly nonlinear) to the varying of QD radius for the small radii, and almost not sensitive for the big QD radii.

Finally, the main conclusion is that for the nanosize QDs, the shifts of electron and hole ground levels are created by the interaction of these quasiparticles with L- and I-phonons due to all the states of discrete and continuous spectrum. For the small QDs, the shifts of ground energy levels have a strong nonlinear dependence and for the big QDs, they almost do not depend on QD radius and have the magnitude close to the shifts of ground levels in a massive crystal creating QD. Due to different effective masses of light and heavy holes, the splittings of their ground levels are complicated functions of $\mathrm{QD}$ radius and $\mathrm{Al}$ concentration in $\mathrm{Al}_{x} \mathrm{Ga}_{1-x} \mathrm{As}$ medium.

\section{References}

1. Ercelebi A., Senger R.T. Ground-state description of quasi-one-dimensional polarons with arbitrary electron-phonon coupling strength. // Phys. Rev. B, 1996, vol. 53, p. $11008-11015$.

2. Huang K., Zhu B.F. Dielectric continuum model and Frohlich interaction in superlattices. // Phys. Rev. B, 1988, vol. 38, p. 13377-13382.

3. Hai G.Q., Peeters F.M., Devreese J.T. Polaron energy and effective mass in a quantum well. // Phys. Rev. B, 1991, vol. 42, p. 11063-11070.

4. Wong X.F., Lei X.L. Polar-optic phonons and high-field electron transport in cylindrical GaAs/AlAs quantum wires. // Phys. Rev. B, 1994, vol. 49, p. 4780-4785.

5. Li X.Q., Arakawa Y. Confined optical phonons in semiconductor quantum dots. // Solid State Commun., 1999, vol. 109, p. 351-356.

6. Mori N., Ando T. Electron-optical-phonon interaction in single and double heterostructures. // Phys. Rev. B, 1988, vol. 40, p. 6175-6182.

7. Stroscio M.A., Kim K.W., Littlejohn M.A., Chnang H. Polarization eigenvectors of surface-optical phonon modes in a rectangular quantum wire. // Phys. Rev. B, 1990, vol. 42 , p. $1488-1495$.

8. Knipp P.A., Reineke T.L. Interface phonons of quantum wires. // Phys. Rev. B, 1992, vol. 45, p. 9091-9098.

9. Klein M.C., Hache F., Ricard D., Flytzanis C. Size-depedence of electron-phonon coupling in semiconductor nanospheres: The case of CdSe. // Phys. Rev. B, 1990, vol. 42, p. 11123-11132.

10. Nomura S., Kobalayashi T. Exciton-Lo-phonon couplings in spherical semiconductor microcrystallites. // Phys. Rev. B, 1992, vol. 45, p. 1305-1315.

11. Marini J.C., Stebe B., Kartheuser E. Exciton-phonon interaction in CdSe and $\mathrm{CuCl}$ polar semiconductor nanospheres. // Phys. Rev. B, 1994, vol. 50, p. 14302-14311.

12. Tanatar B., Guven K., Bennett C.R., Constantinou N.C. Screening effects on the confined and interface polarons in cylindrical quantum wires. // Phys. Rev. B, 1996, vol. 53, p. 10866-10875.

13. Hai G.Q., Peeters F.M., Devreese J.T. Electron optical-phonon coupling in $\mathrm{GaAs} / \mathrm{Al}_{x} \mathrm{Ga}_{1-x}$ As quantum well due to interface, slab and half-space modes. // Phys. Rev. B, 1994, vol. 49, p. 4780-4785. 
14. Tkach M. Electron-phonon interaction in quantum well quantum dot. // Fiz. tverd. tela, 1997, 39, p. 1109-1115 (in Russian).

15. Abrikosov A.A., Gor'kov L.P., Dzeloshinsky I.E. Methods of Quantum Field Theory in Statistical Physics. Moscow, Fizmatgiz., 1962 (in Russian).

16. Agranovich V.M. Theory of Excitons. Nauka., 1968.

\title{
Взаємодія електронів та дірок з фононами у квантовій точці, що вміщена в напівпровідникове середовище (GaAs/Al $\left.\mathrm{Ga}_{1-x} \mathrm{As}\right)$
}

\author{
М.В.Ткач, М.Я.Міхальова, О.М.Войцехівська, \\ Р.Б.Фартушинський
}

Чернівецький національний університет, 58005 Чернівці, вул. Коцюбинського, 2

Отримано 24 травня 2001 р.

У роботі виконано аналітичний і чисельний розрахунки перенормування L- та I-фононами електронного та діркового спектрів з повним врахуванням конфігураційної взаємодії у квантовій точці, що вміщена в напівпровідникове середовище. Конкретний розрахунок виконано для гетеросистеми $\mathrm{GaAs} / \mathrm{Al}_{x} \mathrm{Ga}_{1-x}$ As.

Установлено, що зсуви основних рівнів електрона та дірки формуються взаємодією цих квазічастинок як з L-, так і з І-фононами за участю всіх станів дискретного та неперервного спектрів. При малих розмірах КТ зсуви основних енергетичних рівнів квазічастинок мають сильно нелінійну залежність, а при великих радіусах КТ вони практично не залежать від розміру КТ та близькі за величиною до зсувів основних рівнів у масивному кристалі, з якого утворена КТ. Через різницю ефективних мас важкої та легкої дірок розщеплення іх основних рівнів має складну залежність від радіуса КТ та концентрації Al (x) у середовищі $\mathrm{Al}_{x} \mathrm{Ga}_{1-x} \mathrm{As}$.

Ключові слова: наногетеросистема, взаємодія, електрон, дірка, фонон

PACS: $79.60 . J v, 63.20 . D j$ 
